\title{
Primary Tracheal Small Cell Carcinoma Treated by Concurrent Chemoradiotherapy
}

\author{
June Hong Ahn, Jin Hong Chung, Kwan Ho Lee, Kyeong Cheol Shin, Eun Young Choi, Hyun Jung Jin \\ Department of Internal Medicine, Yeungnam University College of Medicine, Daegu, Korea
}

\begin{abstract}
Primary tracheal tumors are rare and present with signs and symptoms of airway obstruction, and thus, are often initially diagnosed as asthma or chronic obstructive lung disease at an early stage. For this reason, many patients present with advanced stage disease at initial diagnosis. The authors report the case of a 73-year-old male heavy smoker with dyspnea and stridor. Chest computed tomography showed a tracheal mass, and he received a diagnosis of primary tracheal small cell carcinoma after bronchoscopic biopsy. Concurrent chemoradiotherapy was performed and after 5 years of outpatient clinic follow-up he continued to do well with no evidence of disease recurrence.
\end{abstract}

Keywords: Small cell carcinoma; Trachea; Chemoradiotherapy

\section{INTRODUCTION}

Small cell carcinoma (SCC) was first described in the lung, and is commonly of pulmonary origin. Small cell lung cancer (SCLC) is a distinct clinical entity as it has a characteristic biology and histology. Extrapulmonary small cell carcinoma (EPSCC) can arise in multiple organs sites and constitutes the rare clinicopathologic entity of aggressive, high-grade neuroendocrine tumors. EPSCC represents $2.5 \%$ to $5 \%$ of all SCCs and the most predominant sites are the gastrointestinal tract, the genitourinary tract, head and neck, breast, thymus, and an unknown primary site.

EPSCC is often confused with metastatic SCLC, but primary tracheal SCC and primary SCLC with metastatic disease are different clinical entities. In particular, EPSCC is usually fatal, and has 5-year survival rate of less than $15 \%$. We report a rare case of primary tracheal SCC that was successfully treated by concurrent chemoradiotherapy (CCRT).

\section{CASE REPORT}

A 73-year-old self-employed man who was a current smoker (one pack of cigarettes per day for 40 years) visited outpatient clin- ic of Yeungnam University Medical Center with a 10-day history of dyspnea and hoarseness. He had hypertension and old tuberculosis and had history of decompressive laminectomy performed 8 years previously for tuberculous spondylitis. Initial examination revealed inspiratory stridor and chest X-ray revealed narrowing of the tracheal air column at the T2-3 level with intraluminal soft tissue mass density along the right side wall (Fig. 1). The patients was alert, and afebrile, and his respiration was stable (20 breaths/min), but he was tachycardic (110 beats/min).

The laboratory results were as follows: leukocyte count $5.59 \mathrm{k} / \mu$ $\mathrm{L}$, hemoglobin $15.3 \mathrm{~g} / \mathrm{dL}$, and platelets $307 \mathrm{k} / \mu \mathrm{L}$. Liver and renal function and electrolytes were within normal ranges. Arterial blood gas analysis revealed $\mathrm{pH}$ 7.414, $\mathrm{PaO}_{2} 83.6 \mathrm{~mm} \mathrm{Hg}, \mathrm{PaCO}_{2}$ $39.8 \mathrm{~mm} \mathrm{Hg}, \mathrm{HCO}_{3}-24.9 \mathrm{mmol} / \mathrm{L}$, and $\mathrm{SaO}_{2} 97.7 \%$ in room air.

Contrast-enhanced chest computed tomography (CT) revealed a lobulated mass at the proximal trachea extending to the larynx (Fig. 2A, B). Old tuberculosis scarring was observed at both upper lobes. No evidence of metastatic lymphadenopathy or of a lung field mass was observed, and no abnormalities were detected in great vessels, liver, or adrenal glands.

Fiberoptic bronchoscopy showed a $4.2 \times 3.1-\mathrm{cm}$-sized lobulated, irregularly surfaced mass at the upper trachea with near-total oc-
Correspondence to: Jin Hong Chung

Department of Internal Medicine, Yeungnam University College of Medicine, 170 Hyeonchung-ro, Nam-gu, Daegu 42415, Korea

Tel: +82-53-620-3840, Fax: +82-53-623-8201, E-mail: jhchn@med.yu.ac.kr

Received: May 11, 2016 / Accepted after revision: Sep. 6, 2016
(C) 2016 Soonchunhyang Medical Research Institute This is an Open Access article distributed under the terms of the Creative Commons Attribution Non-Commercial License (http://creativecommons.org/licenses/by-nc/4.0/). 


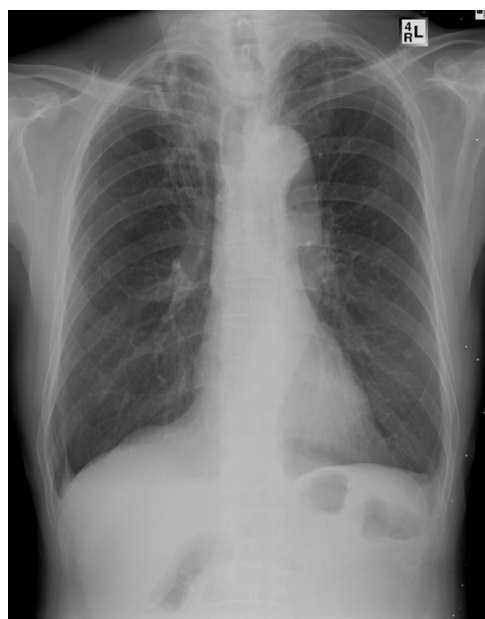

Fig. 1. Plain chest radiography showed narrowing of tracheal lumen with intraluminal soft tissue mass density. clusion $5 \mathrm{~cm}$ above the carina (Fig. 2C). A biopsy was performed, and subsequent routine hematoxylin and eosin staining revealed diffuse sheets of small, blue, round cell tumor with cells two to three times larger than that of mature lymphocytes. Extensive necrotic background was present. Cytoplasm was sparse, and nuclear features included finely granular chromatin without prominent nucleoli, nuclear molding, and marked crushing artifact, which were highly suggestive of SCC (Fig. 3).

Multiple immunohistochemical (IHC) stainings were performed to confirm the diagnosis. Stainings for CD56, and synaptophysin (both neuroendocrine markers) were positive. Staining for thyroid transcription factor-1 (TTF-1) was also positive (Fig. 4). Hematoxylin and eosin staining with IHC staining supported a diagnosis of SCC.
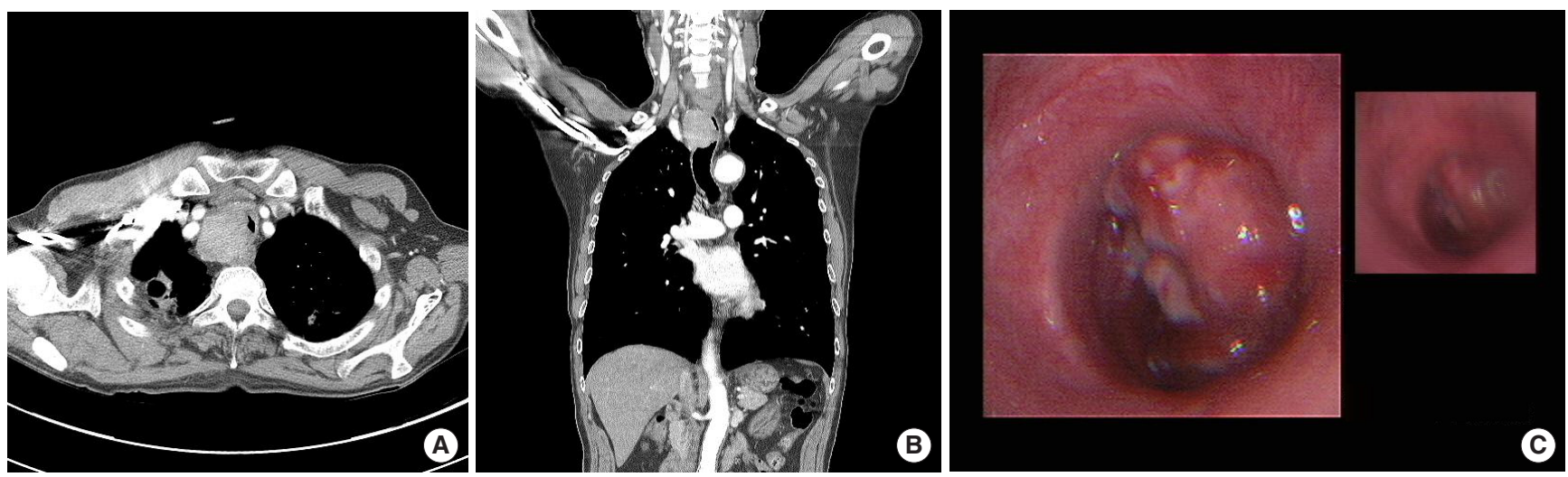

Fig. 2. Chest computed tomography (A: transverse view, B: coronal view) showing a lobulated mass at the proximal trachea (C) Bronchoscopy revealed a 4.2×3.1-cmsized lobulated, irregularly surfaced mass at the upper trachea causing near-total occlusion $5 \mathrm{~cm}$ above the carina.


Fig. 3. Histopathological findings. The tumor showed small, blue, round tumor cells, with scant cytoplasm, finely granular chromatin, and no prominent nucleoli, and showed marked crushing artifact indicative of small cell carcinoma. (A) $H \& E, \times 100$. (B) $H \& E, \times 200$. 

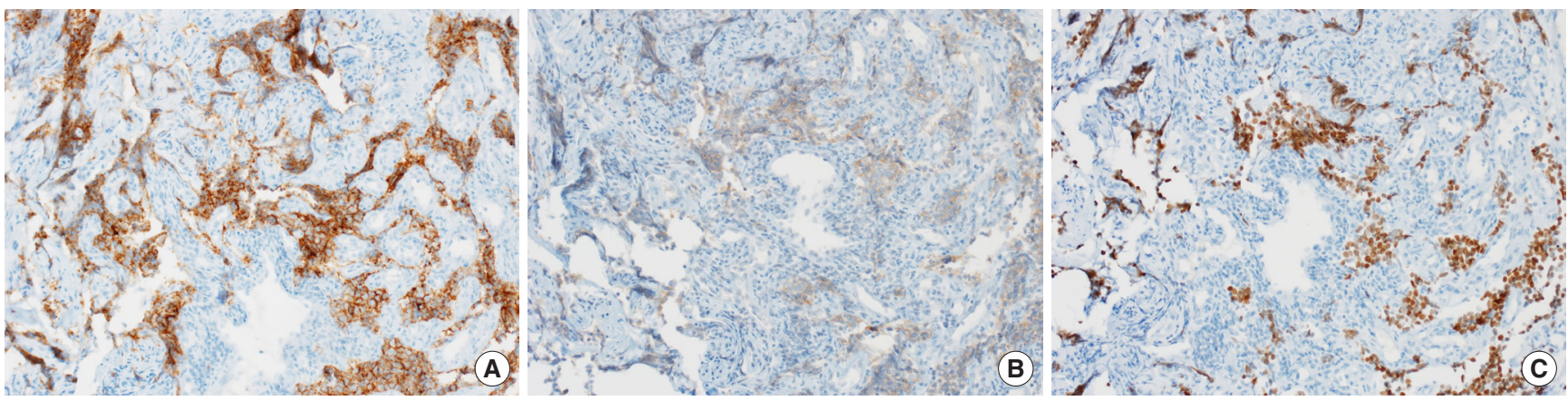

Fig. 4. The tumor cells were immunohistochemically reactive for CD56 (A: IHC staining, $\times 200)$, synaptophysin (B: IHC staining, $\times 200)$, and thyroid transcription factor-1 (C: IHC staining, × 200). IHC, immunohistochemical.


Fig. 5. After radiotherapy and 2 cycles of chemotherapy, chest computed tomography (A: transverse view, B: coronal view) showed no visible tumor. (C) Bronchoscopy performed after radiotherapy and 2 cycles of chemotherapy revealed only tracheal mucosa ulceration and no fistula or tracheal tumor.

Further studies were performed before treatment for cancer staging. Brain magnetic resonance imaging (MRI) revealed no brain metastasis, and a bone scan revealed no bone metastasis. Positive emission tomography-computed tomography (PET-CT) revealed no nodal or distant metastasis.

Surgery was not performed, instead the patient received 6 cycles of systemic chemotherapy $\left(100 \mathrm{mg} / \mathrm{m}^{2}\right.$ of etoposide and $80 \mathrm{mg} / \mathrm{m}^{2}$ of cisplatin every 3 weeks), and concurrent radiotherapy $(3,000$ cGy in 10 fractions) to the tracheal mass.

After radiotherapy and 2 cycles of chemotherapy, chest CT and bronchoscopy was performed to evaluate response to chemotherapy. Chest CT showed complete remission (Fig. 5A, B). Bronchoscopy revealed only tracheal mucosa ulceration without evidence of fistula or of the tracheal tumor (Fig. 5C). The patient was subsequently administered four additional cycles of systemic chemotherapy. Five years after treatment completion, the patient was well with no evidence of recurrence.

\section{DISCUSSION}

Primary tracheal tumors are rare and their incidence is much lower than those of laryngeal or bronchial cancer. The annual incidence of tracheal cancer is about 0.14 per 100,000 persons per year, and it accounts for only $0.2 \%$ of all tumors of the respiratory tract and only $0.02 \%$ to $0.04 \%$ of all malignant diseases [1-3].

Patients often present with symptoms of central-airway obstruction, such as dyspnea, cough, and wheezing. Patients with tracheal tumors are frequently misdiagnosed as having asthma or chronic obstructive pulmonary disease (COPD). Diagnosis is commonly delayed, and many patients present with advanced disease. Therefore, strong suspicion is required if tracheal tumors are to be diagnosed at an early stage [1].

Primary tracheal tumors can arise from surface epithelium, salivary glands, or mesenchyme of the trachea. In adults, $90 \%$ of primary tracheal tumors are malignant, whereas only $10 \%$ to $30 \%$ are malignant in children [1]. Squamous cell carcinoma is the pre- 
dominant tumor type and adenoid cystic carcinoma is the second most common type. Neuroendocrine tumors, including SCC, account for $9.7 \%$ of all primary tracheal tumors [4].

Primary tracheal SCC is a type of EPSCCs, which are extremely rare and can originate from multipotent stem cells native to all tissues. EPSCCs have most commonly been described in the gastrointestinal tract, genitourinary tract, and head and neck region. Sometimes SCC will occasionally present with metastatic disease, and the primary site cannot be identified (SCC of unknown primary) [5].

The initial evaluation of a patient with primary tracheal SCC should include assessments of the primary tumor and regional lymph nodes to assess the extent of locoregional disease. Chest CT is needed to detect possible primary SCLC. Bronchoscopy is commonly used to achieve a diagnosis and stage tracheal tumors based on appearance and subsequent pathologic findings. PET-CT is useful for staging and evaluating treatment response. Brain MRI can be considered if neurologic symptoms are present. Based on the results of such evaluations, disease confined to a primary site and regional nodes is classified as limited disease, while all others are classified as extensive disease [5].

SCC fits into the spectrum of neuroendocrine tumors as a highgrade tumor along with large cell neuroendocrine carcinoma and as a low-grade typical carcinoid and intermediate-grade atypical carcinoid. In our case, multiple IHC stainings (positive for CD56 and synaptophysin) suggest neuroendocrine tumors. Hematoxylin and eosin staining showed diffuse sheets of small, blue, and round cell tumor. Cytoplasm was sparse, and nuclear features included finely granular chromatin without prominent nucleoli, nuclear molding, and marked crushing artifact. Extensive necrotic background was present. Staining for TTF-1 was also positive. These findings were consistent with SCC [6].

EPSCC should not be confused with metastatic SCLC, as it is a clinical entity distinct from SCLC. Because of the rarity of EPSCC, management of patients is patterned after that for other tumor types arising in the same extrapulmonary sites. Despite aggressive locoregional treatment with surgery, relapse is common and adjuvant systemic chemotherapy is generally recommended. In most patients with limited disease, primary treatment by CCRT can be as effective as surgery. No large randomized clinical trial has been undertaken to guide the treatment of patients with EPSCC due to its rarity and diverse origins [7]. Generally a platinum-based regi- men with etoposide is used. The prognosis for patients with disseminated disease is poor despite CCRT and is similar to that for patients with extensive SCLC [5]. Particularly in patients with tracheal SCC, endotracheal debridement and endotracheal stents are also therapeutic options in patients with tracheal obstruction by tumors. Endotracheal stents are reliable and durable palliative methods in $80 \%$ to $90 \%$ of properly-selected tracheal tumor patients $[1,8]$.

Prophylactic cranial irradiation is not commonly recommended in primary tracheal SCC because the incidence of brain metastasis is lower in EPSCC than in SCLC. However, EPSCCs with a primary origin in the head, neck, or prostate are exceptions because they are associated with higher incidences of brain metastasis and thus, prophylactic cranial radiation can be considered in such cases [4].

EPSCC is usually a fatal disease, with a 5-year survival rate of only $13 \%$ [7]. In our patient, early diagnosis and aggressive CCRT achieved a favorable outcome without any clinical complications. The patient continues to do well without any evidence of recurrence at more than 5 years after diagnosis.

In conclusion, primary tracheal tumors present with signs and symptoms of airway obstruction, and thus, are often misdiagnosed as asthma or COPD at an early stage. For this reason, many patients present with advanced stage disease at initial diagnosis. Hence, clinicians should consider chest CT or bronchoscopy in a patient with uncontrolled asthma or COPD to evaluate tumor in central airways.

\section{REFERENCES}

1. Macchiarini P. Primary tracheal tumours. Lancet Oncol 2006;7:83-91.

2. Honings J, van Dijck JA, Verhagen AF, van der Heijden HF, Marres HA. Incidence and treatment of tracheal cancer: a nationwide study in the Netherlands. Ann Surg Oncol 2007;14:968-76.

3. Junker K. Pathology of tracheal tumors. Thorac Surg Clin 2014;24:7-11.

4. Urdaneta AI, Yu JB, Wilson LD. Population based cancer registry analysis of primary tracheal carcinoma. Am J Clin Oncol 2011;34:32-7.

5. Walenkamp AM, Sonke GS, Sleijfer DT. Clinical and therapeutic aspects of extrapulmonary small cell carcinoma. Cancer Treat Rev 2009;35:22836.

6. Travis WD. Update on small cell carcinoma and its differentiation from squamous cell carcinoma and other non-small cell carcinomas. Mod Pathol 2012;25 Suppl 1:S18-30.

7. Galanis E, Frytak S, Lloyd RV. Extrapulmonary small cell carcinoma. Cancer 1997;79:1729-36.

8. Wood DE. Management of malignant tracheobronchial obstruction. Surg Clin North Am 2002;82:621-42. 